\title{
Comparison of different breath-by-breath gas exchange algorithms using a gas exchange simulation system
}

\author{
Maria Pia Francescato ${ }^{\mathrm{a}, *}$, Lutz Thieschäfer ${ }^{\mathrm{b}}$, Valentina Cettolo ${ }^{\mathrm{a}}$, Uwe Hoffmann ${ }^{\mathrm{b}}$

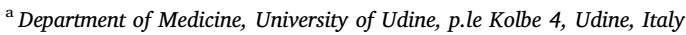 \\ ${ }^{\mathrm{b}}$ Institute of Physiology and Anatomy, German Sport University Cologne, Am Sportpark Müngersdorf 6, Cologne, Germany
}

\section{A R T I C L E I N F O}

\section{Keywords:}

Gas exchange

Breath-by-breath calculation algorithms

Gas stores

Thermodynamic conversions

\begin{abstract}
A B S T R A C T
Background: Mechanical Gas Exchange Simulation Systems (GESS) have never been used to compare different breath-by-breath oxygen uptake calculation algorithms.

Methods: Oxygen uptakes were calculated for each GESS cycle by the "Expiration-only" algorithm (estimating inspiratory volume from the expiratory one), and by two "alveolar" algorithms (both processing inspiratory and expiratory flows and designed to account for the changes in lung gas stores). The volume of oxygen stored in the GESS from one cycle to the subsequent one was either maintained constant or increased/decreased by changing the pumped gas volumes.

Results: Overlapping oxygen uptakes were obtained maintaining constant the volume of oxygen stored (grand average: $0.420 \pm 0.019 \mathrm{~L} / \mathrm{min}, \mathrm{p}=\mathrm{ns}$ ). The "Expiration-only" algorithm overestimated the decreases of the stored oxygen by 34\%, whereas the "alveolar" algorithms underestimated the increases by $25 \%$; in the other conditions, the changes of the stored oxygen were appropriately accounted for.

Conclusions: The use of "alveolar" algorithms is recommended, particularly so when abrupt changes in the stored oxygen volume occur.
\end{abstract}

\section{Introduction}

Measurement of breath-by-breath gas exchange in humans is becoming increasingly common in research laboratories and medical centers. Automatic metabolic units are frequently used, with the underlying assumption that their measurement errors fall within acceptable limits. Variations of precision can be suitably detected by a mechanical device that simulates metabolic gas exchange (Ward, 2018), i.e. using a Gas Exchange Simulation System (GESS). Indeed, this kind of devices generates respiratory cycles having predetermined and known characteristics, while guaranteeing reproducibility. So far, these devices have been used to evaluate hardware components of the gas exchange measurement devices (Prieur et al., 2003; Rodriguez et al., 2008), while a GESS can be appropriately used also to compare the performance of different gas exchange calculation algorithms.

The simplest algorithm for the determination of breath-by-breath gas exchange on the basis of flow and gas traces acquired at the mouth, is the computation of the difference between the inspired and the expired gas volumes within the same breath. To avoid the extreme erratic results obtained with this method (Auchincloss et al., 1966), different algorithms have been developed to calculate breath-by-breath gas exchange (Auchincloss et al., 1966; Beaver et al., 1973; Busso and Robbins, 1997; Cettolo and Francescato, 2018; Grønlund, 1984; Swanson, 1980; Wessel et al., 1979). All of them share the concept that only oxygen and carbon dioxide are exchanged and that there is no net exchange of the remaining gases (overall defined as nitrogen), although the volume of the latter does not have to be null for the data processing with the algorithms. In addition, most of the algorithms were designed, theoretically, to take account of the changes in the volume of gas stored in the lungs and, since they are believed to estimate the gas exchange at the alveolar-to-capillary membrane, they often are defined as "alveolar" algorithms (Golja et al., 2018). All the above algorithms have been tested essentially under moderate intensity exercise. Nevertheless, a validation under conditions of well-known breathing patterns, as those that can be created with a GESS, can provide useful information concerning the specific characteristics of each algorithm, in particular when the "alveolar" algorithms are to be compared with the remaining ones; differences in the $\mathrm{O}_{2}$ uptake values yielded by the various algorithms should emerge when accounting for the changes in gas stores, induced by changes in volume and/or end-expiratory gas fractions from one cycle to the subsequent one.

The aim of the present work was the comparison of the performance

\footnotetext{
* Corresponding author at: Department of Medicine, University of Udine, P.le Kolbe 4, 33100, Udine, Italy.

E-mail address: mariapia.francescato@uniud.it (M.P. Francescato).
} 


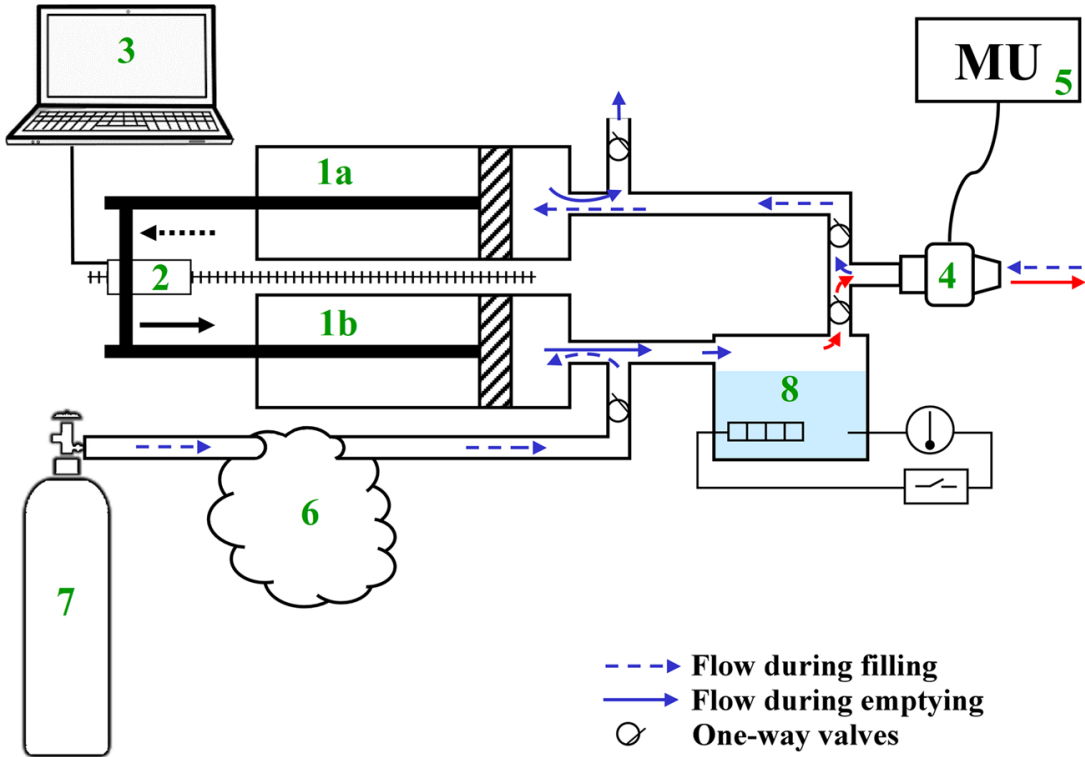

Fig. 1. Schematic representation of the Gas Exchange Simulation System (GESS) used in the present investigation together with the signals acquisition system.

1. syringes ( $a$ and $b$ );

2. servomotor generating the synchronous movements of the pistons along the gantry module;

3. computer controlling the motor;

4. support and sensors of the metabolic unit for assessment of flow and gas fractions;

5. metabolic unit;

6. polyethylene bag;

7. supply cylinder, containing gas with known composition;

8. thermostatic water bath. of gas exchange algorithms that provide data in real-time, namely, the "Expiration-only" (EXP) (Beaver et al., 1973; Ward, 2018), the "Wessel" (WES) (Wessel et al., 1979), and the "Independent breath" (IND) (Cettolo and Francescato, 2018) algorithms, respectively. Comparison was also performed with data obtained by simply integrating flow and gas fractions ("Mouth" algorithm) (Wessel et al., 1979). A regular breathing pattern was simulated, as well as different abrupt changes of the gas volumes stored in the system were inserted, in order to provide proof of concept of the effectiveness of the algorithms under investigation. A GESS with the advanced feature allowing to heat and humidify the gas of the expired flow, so far not implemented in other devices, was used.

\section{Methods}

\subsection{The gas exchange simulation system}

The GESS used in this investigation (Fig. 1) was an enhanced version of a previously used device (Rodriguez et al., 2008). It is composed of two parallel syringes (of $3 \mathrm{~L}$ volume each; $1 \mathrm{a}, 1 \mathrm{~b}$ ), whose pistons were moved synchronically along a gantry module by a servomotor (2), in turn controlled by a computer (3). The nozzles of the syringes were connected, by means of a system of tubes and one-way valves (to predetermine the direction of flow), to the support (4) of the sensors assessing flow and gas fractions.

The sensors of the metabolic unit (5) measured a) a simulated inspiratory flow of ambient air during the filling of the syringes, b) a simulated expiratory flow of gas with known composition during the emptying of the syringes. The desired volume of the gas with known composition was drawn from a gas container (60 liters non-diffusing gas bag; 6), continuously filled from a gas cylinder (7). A thermostatic water bath (8) was positioned between the syringe $1 \mathrm{~b}$ and the support of the metabolic unit sensors (4), allowing the heating $\left(37^{\circ} \mathrm{C}\right)$ and water vapor saturation of the gas.

The supply cylinder contained a gas mixture with $16.00 \% \mathrm{O}_{2}, 4.99 \%$ $\mathrm{CO}_{2}$ and $\mathrm{N}_{2}$ as balance; the chosen gas mixture allowed us to have equal $\mathrm{N}_{2}$ fractions in the moved gases from both syringes.

The experimental protocol was performed at least twice to check for the overall stability of the experimental instrumentation, composed of the GESS and of the metabolic unit; the pertinent coefficient of variations (CV) were evaluated. The volumes of gas measured by the flow sensor of the metabolic unit during the filling or emptying of the syringes showed a CV $<0.8 \%$. The overall $\mathrm{CV}$ of the $\mathrm{N}_{2}$ fraction (over both the filling and the emptying of the syringes) was $<0.2 \%$ and the end-expiratory gas fractions showed a $\mathrm{CV}<0.5 \%$ for both $\mathrm{O}_{2}$ and $\mathrm{CO}_{2}$.

\subsection{Experimental protocol}

The breathing pattern was simulated in the GESS imposing sinusoidal changes in the position of the pistons of the syringes as a function of time.

Series of identical cycles (composed of one filling and the subsequent emptying stroke) were generated yielding a moved volume of $1.0 \mathrm{~L}$ per stroke with a frequency of 12 cycles per minute ("regular" cycles). Interspersed among the "regular" cycles, maintaining constant the frequency, "aberrant" strokes were introduced by changing the moved volume so as to obtain a change of the volume of gas contained in the syringes from one cycle to the subsequent one. Four different kinds of "aberrant" strokes were generated, namely, increases of $+0.5 \mathrm{~L}$, $+1.0 \mathrm{~L}$, or $+1.5 \mathrm{~L}$, and a decrease of $-0.3 \mathrm{~L}$ from the regular volume of $1.0 \mathrm{~L}$. The "aberrant" strokes were inserted:

a) in the filling stroke only;

b) in the emptying stroke only;

c) in both strokes, consecutively, in the filling/emptying sequence;

d) in both strokes, consecutively, in the emptying/filling sequence; this is tantamount saying that the "aberrant" stroke was inserted in the emptying stroke only of the cycle and inserted in the filling stroke only of the subsequent cycle.

\subsection{Assessment of ventilatory parameters}

Flow, $\mathrm{O}_{2}$ and $\mathrm{CO}_{2}$ fractions $\left(\mathrm{FO}_{2}\right.$ and $\mathrm{FCO}_{2}$, respectively) were continuously recorded using a Metalyzer 3B metabolic unit (Cortex, Leipzig, Germany). Before each measurement session, according to the instructions of the manufacturer, the turbine was calibrated by means of a 3 liters syringe (Cortex, Leipzig, Germany) and the analyzers were calibrated with a gas mixture of known composition $\left(\mathrm{FO}_{2}=16.0 \%\right.$; $\mathrm{FCO}_{2}=4.0 \% ; \mathrm{FN}_{2}$ as balance) and ambient air. The software operating the metabolic unit allowed the recording of gas fractions and flow signals as text files with a sampling frequency of $50 \mathrm{~Hz}$. Traces of gas fractions and flow were synchronized by the firmware of the metabolic unit, which also converted flow to BTPS conditions. Fig. 2, panel A, illustrates an example of the recorded traces during a few "regular" cycles.

For each stroke throughout the acquisitions, inspired $\left(\mathrm{V}_{\mathrm{I}}\right)$ and/or 

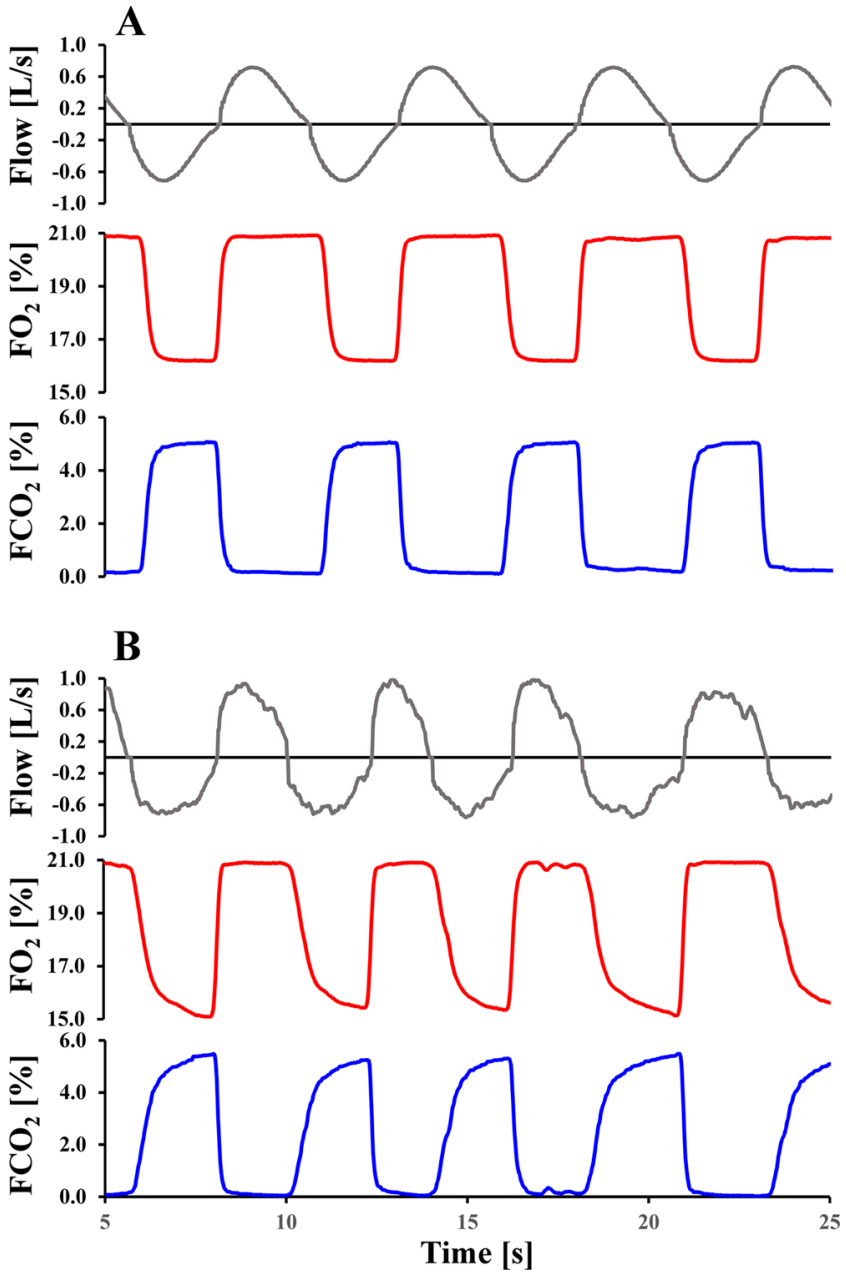

Fig. 2. Examples of the flow and $\mathrm{O}_{2}$ and $\mathrm{CO}_{2}$ fraction traces recorded by means of the metabolic unit from the GESS during "regular" cycles (panel a) and during light intensity exercise in a volunteer (panel b).

Despite the striking similarities between the simulated breathing and the human data, the figure shows that human inspiration and expiration show an asymmetry either in the duration and in the behaviour of the waveform, and that the human fraction traces have a drift during the final part of the expirations, that does not appear during the emptying of the syringes, simulating the expirations. In both panels, positive flow corresponds to inspiration, negative one corresponds to expiration, respectively, as interpreted by the metabolic unit.

expired $\left(\mathrm{V}_{\mathrm{E}}\right)$ gas volumes were calculated from the original flow trace as integrals between two successive changes in flow direction; cycle frequency $\left(b_{\mathrm{f}}\right)$ was calculated as the reciprocal of the time elapsed between two subsequent starts of inspiration; ventilation $\left(\dot{V}_{E}\right)$ was calculated as $\mathrm{V}_{\mathrm{E}} \mathrm{x} \mathrm{b}_{\mathrm{f}}$.

Breath-by-breath oxygen uptake $\left(\dot{V} \mathrm{O}_{2}\right)$ was calculated from the same original gas fractions and flow traces, as yielded by the metabolic unit, by means of all the algorithms under investigation, i.e., the "Independent breath" ( $\dot{V} \mathrm{O}_{2}^{I N D}$; (Cettolo and Francescato, 2018)), as well as the "Wessel" ( $\dot{V} O_{2}^{W E S}$; (Wessel et al., 1979)), and the "Expiration only" $\left(\dot{V} O_{2}^{E X P}\right.$; (Ward, 2018)) algorithms, respectively; in addition, oxygen uptake data were calculated also by simple integration of flow and gas fractions ("Mouth" algorithm; $\dot{V} O_{2}^{M}$; (Wessel et al., 1979)). Computerized procedures were specifically developed in C language under the Unix-like Cygwin environment (Red Hat Cygwin, version 4.3.39). See the Appendix A for the mathematical description of the algorithms under investigation.

The value of one minus the sum of measured $\mathrm{O}_{2}$ and $\mathrm{CO}_{2}$ fractions was labeled $\mathrm{FN}_{2}$ being essentially composed of nitrogen.
All the calculated oxygen volumes and uptakes were expressed in STPD conditions; flow and ventilation were in BTPS conditions.

\subsection{Data analysis}

The effectiveness of the algorithms under investigation to account for the changes in gas volume stored in the GESS from one cycle to the following one was evaluated as follows. The changes in the stored volume of $\mathrm{O}_{2}\left(\Delta \mathrm{VO}_{2 s}\right)$ induced by the "aberrant" strokes were calculated theoretically and compared to the changes estimated through the $\dot{V} \mathrm{O}_{2}$ yielded by the different algorithms for the corresponding "aberrant" cycles.

The "theoretical" $\Delta \mathrm{VO}_{2 s t}$ were calculated on the basis of the changes of the moved gas volumes in the "aberrant" cycles as follows:

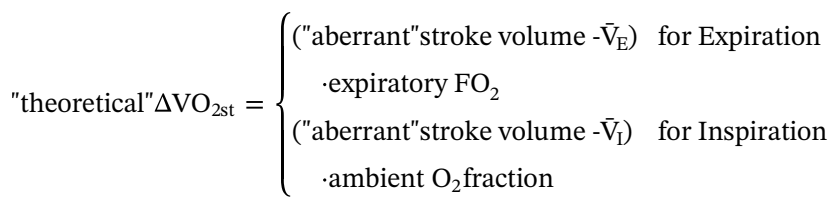

where $\bar{V}_{E}$ and $\bar{V}_{I}$ are the average expiratory and inspiratory volumes of the regular strokes, expressed in STPD conditions; the ambient $\mathrm{O}_{2}$ fraction was set to 0.2095 and the expiratory $\mathrm{O}_{2}$ fraction was set to the average value of the corresponding simulated expiration. Of note, the "theoretical" $\Delta \mathrm{VO}_{2 s t}$ has a meaning only if at least one "aberrant" stroke occurred within the cycle.

The changes in the amounts of $\mathrm{O}_{2}$ accounted for by a specific algorithm, as estimated through its $\dot{V} \mathrm{O}_{2}$, have been calculated for each "aberrant" cycle as follows:

"accounted" $\Delta \mathrm{VO}_{2 \mathrm{st}}=$ "theoretical" $\Delta \mathrm{VO}_{2 \mathrm{st}}-\left(\left(\frac{\dot{\mathrm{VO}}}{\mathrm{b}_{\mathrm{f}}}\right)_{\text {"aberrant"cycle }}-\dot{\mathrm{V}} \mathrm{O}_{2} \mathrm{~b}_{\mathrm{f}}\right)$

where $\overline{\mathrm{b}_{\mathrm{f}}}$ and $\overline{\mathrm{VO}_{2}}$ are the averages, over all the "regular" cycles, of breath frequency and of the $\dot{V} \mathrm{O}_{2}$ obtained using the specific algorithm.

\subsection{Statistical analysis}

Statistical analyses were performed using the SPSS software (Chicago, Ill., USA); results were expressed as means \pm SD and significance level was set to $\mathrm{p}<0.05$.

Normal distribution of the $\dot{V} \mathrm{O}_{2}$ data yielded by the different algorithms for the "regular" cycles was confirmed by the Shapiro-Wilk test. A repeated measure Multivariate Analysis of Variance (MANOVA) was used to detect differences among the 4 investigated algorithms (Algorithm effect). The Mauchly's test was used to check if the sphericity assumption appeared to be violated; if the Mauchly's test was significant, the Huynh-Feldt $\varepsilon$ was used to adjust the degrees of freedom. Post-hoc difference contrast was used to detect significant differences inside the within subjects effects.

Correlation between variables was assessed by means of the Pearson's correlation coefficient and the least-squares method was applied to calculate the slopes and intercepts of the regression lines, together with the corresponding widths of the $95 \%$ confidence intervals and statistical significance levels against the identity line (i.e. slope $=1$ and intercept $=0$ ).

\section{Results}

The complete flow trace of one acquisition is illustrated in Fig. 3. The "aberrant" strokes, corresponding to the changes of $+1.0 \mathrm{~L},+0.5 \mathrm{~L}$, $+1.5 \mathrm{~L},-0.3 \mathrm{~L}$, compared to the regular moved volume, respectively, are clearly visible as spikes of deeper/shallower inspiratory (positive) or expiratory (negative) flow. The corresponding ventilation, according to its classical definition, showed spikes where the cycles were performed with an "aberrant" expiratory stroke; conversely, ventilation 


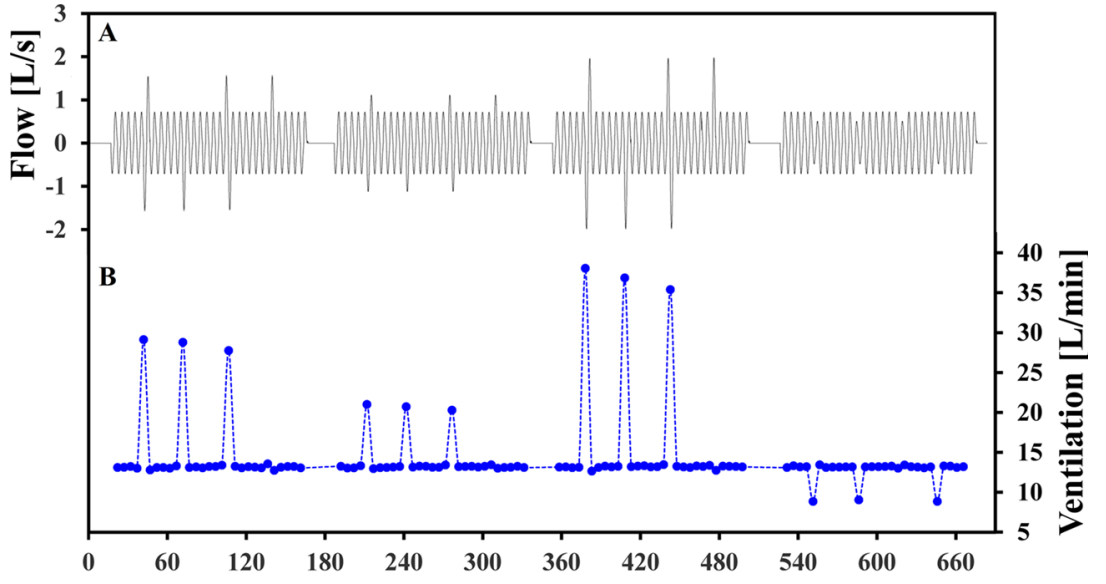

Fig. 3. Acquired flow trace (panel a) and calculated ventilation (panel b) throughout the acquisition period.

The flow trace (continuous line; primary vertical axis) provided by the metabolic unit clearly shows the spikes corresponding to the "aberrant" strokes. The different dimensions of the spikes are related to the different changes imposed to the "regular" stroke volume, and are reflected in a change in expiratory or inspiratory volume. Positive flow corresponds to inspiration, negative one corresponds to expiration, respectively, as interpreted by the metabolic unit.

Ventilation (dots; secondary axis) resulted practically constant for the "regular" strokes; different values are observable for the cycles wherein an "aberrant" expiratory stroke was performed, according to the change in the moved volume. To be noted that ventilation remained quite constant where only the inspiratory strokes were "aberrant".

Both variables are expressed in BTPS conditions.

Time $[\mathbf{s}]$

remained quite constant where only the inspiratory strokes were "aberrant".

Fig. 4 illustrates, for a portion of the recording period, the $\dot{V} \mathrm{O}_{2}$ data calculated using the four algorithms under investigation. Considering only the "regular" cycles, significantly different $\mathrm{VO}_{2}$ values (MANOVA, algorithm effect, $\mathrm{p}<0.001$ ) were obtained; post-hoc contrasts highlighted that only the values obtained with the "Mouth" algorithm were significantly different (difference contrast, $\mathrm{p}<0.001$ ) compared to the values yielded by the remaining three algorithms, which were overlapping (difference contrast, $\mathrm{p}=\mathrm{ns}$ for all the comparisons). Average $\dot{V} \mathrm{O}_{2}$ amounted to $0.474 \pm 0.022 \mathrm{~L} / \mathrm{min}$ for the "Mouth" algorithm and to $0.420 \pm 0.019 \mathrm{~L} / \mathrm{min}$ when expressed as grand-average of all the other three algorithms, respectively.

Different behaviors were observed in the $\dot{V} \mathrm{O}_{2}$ data when the "aberrant" cycles were analyzed; the amplitude of the spikes were modulated by the entity of the change in moved volume, and the reduced volume $(-0.3 \mathrm{~L})$ had an opposite effect compared to the three increased volumes ( $+0.5 \mathrm{~L},+1.0 \mathrm{~L}$ and $+1.5 \mathrm{~L}$, respectively). The data obtained with the IND and WES algorithms showed the same behavior in all the conditions; for these two algorithms, the calculated $\dot{V} \mathrm{O}_{2}$ were not affected when, within the cycle, the expiratory volume only was changed. The $\dot{V} \mathrm{O}_{2}$ yielded by the EXP algorithm were not affected when, within the cycle, the inspiratory volume only was changed. Finally, the data obtained by the "Mouth" algorithm were affected by all the conditions; the changes were larger than those of the other

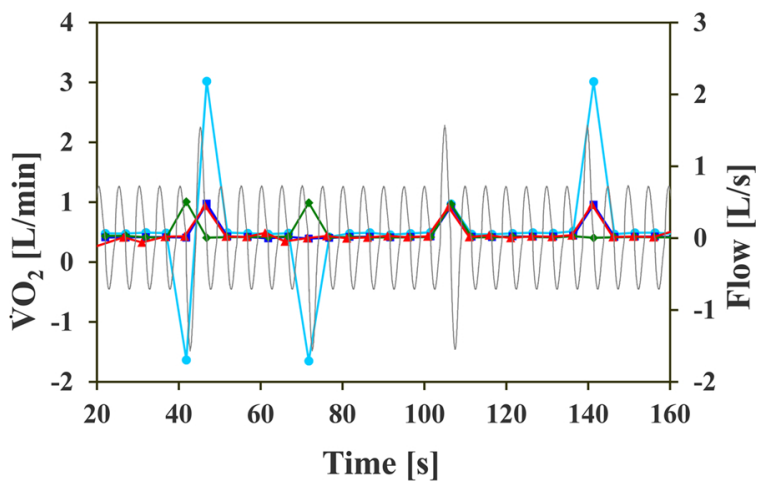

Fig. 4. Oxygen uptake data calculated by means of the four algorithms at stake. The data are illustrated for a portion only of the recording period (in STPD); the corresponding acquired flow (thin line; secondary axis; in BTPS) is also illustrated. Positive flow corresponds to inspiration, negative one corresponds to expiration, respectively, as interpreted by the metabolic unit.

Oxygen uptake data yielded by: "Mouth", $\bullet$ EXP, $\square$ WES, and $\triangle$ IND algorithms, respectively.

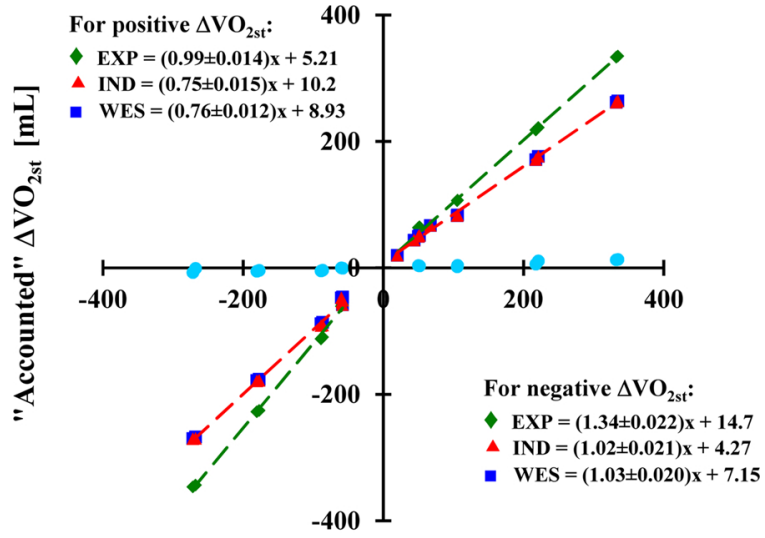

"Theoretical" $\Delta \mathrm{VO}_{2 \mathrm{st}}[\mathrm{mL}]$

Fig. 5. Changes of oxygen volume $\left(\Delta \mathrm{VO}_{2 \mathrm{st}}\right)$ accounted for by a given algorithm. The changes of $\mathrm{O}_{2}$ volume for the "aberrant" breaths are plotted as a function of the theoretical changes in the amount of $\mathrm{O}_{2}$ available in the system (both variables are in STPD). Of note, the positive/negative $\Delta \mathrm{VO}_{2 \text { st }}$ were induced by either increasing/decreasing the filling volume or decreasing/increasing the emptying volume of the syringes.

Changes of oxygen volume accounted for by: • "Mouth", $\triangleleft$ EXP, $\square$ WES, and $\triangle$ IND algorithms, respectively.

algorithms, with the exception of the condition of a deeper inspiration followed by a deeper expiration within the same cycle, where a similar change in $\dot{V} \mathrm{O}_{2}$ was observed for all the algorithms.

Fig. 5 illustrates the changes in the volumes of $\mathrm{O}_{2}$ stored in the GESS accounted for by the algorithms under investigation as a function of the theoretical changes in the volume of $\mathrm{O}_{2}$ stored in the device; the data of all the "aberrant" cycles are illustrated. The "Mouth" algorithm was almost unable to account for the changes of $\mathrm{O}_{2}$ volume stored in the GESS, as suggested by the near zero values observed for all the "aberrant" cycles. The EXP algorithm overestimated the depletion of the $\mathrm{O}_{2}$ volume stored in the GESS, the slope between the theoretical changes and the changes accounted for amounting to $1.339 \pm 0.022$ ( $\mathrm{p}<0.001$ from the identity). In the case of an increase of the stored $\mathrm{O}_{2}$ volume, the EXP algorithm seemed able to completely account for the changes, the slope amounting to $0.985 \pm 0.014$ ( $p=$ ns from the identity), the intercept amounting to $5.206 \pm 2.551 \mathrm{~mL}(\mathrm{p}=\mathrm{ns}$ from 0 ). The IND and WES algorithms showed similar results: the points obtained for the two algorithm were overlapping (Fig. 5). In the case of depleted oxygen stores, the volume changes accounted for by the two algorithms were quite the same as the theoretical change of the stored volume of $\mathrm{O}_{2}$, the slope amounting to $1.020 \pm 0.021(\mathrm{p}=\mathrm{ns}$ from the 
identity), with an intercept of $4.271 \pm 3.381 \mathrm{~mL}(\mathrm{p}=\mathrm{ns}$ from 0 ). Conversely, in the case of an increased stored $\mathrm{O}_{2}$ volume, the two algorithms were able to account for a fairly large portion of the overall change, the slope between the theoretical changes in volume and the volumes accounted for amounting to $0.753 \pm 0.015$ ( $p<0.001$ from the identity).

\section{Discussion}

To our knowledge, this is the first work comparing the performance of different algorithms for the real-time calculation of breath-by-breath oxygen uptake using a Gas Exchange Simulation System (GESS). The main advantage of the use of a GESS is the reproducibility of the simulated breaths, once the parameters determining their characteristics are defined. In addition, the GESS should allow to investigate the changes in the volume of gas stored in the system, splitting the effects induced by the involved components.

Results of the present work showed that, among the four investigated algorithms, the "Mouth" algorithm provided clearly different oxygen uptakes. The remaining three algorithms, designed on different theoretical bases, showed different performances when detecting changes in the volume of oxygen stored in the system as induced by changes of the pumped volumes only.

\subsection{The "aberrant" cycles and the changes in gas stores}

The "aberrant" strokes were inserted among the "regular" cycles in order to change the overall volume of oxygen stored in the device. Indeed, when an "aberrant" cycle included an increased inspiratory volume (or a decreased expiratory one), the stored volume of oxygen was increased; the opposite occurred (i.e. the stored oxygen volume was depleted) for the "aberrant" cycles including an increased expiratory volume or a decreased inspiratory one.

The investigated algorithms showed different behaviors for the "aberrant" cycles, to which corresponded different "accounted" $\Delta \mathrm{VO}_{2 \mathrm{st}}$. Despite the different behavior, the relationships between the theoretical changes in the stored $\mathrm{O}_{2}$ volume and the "accounted" $\Delta \mathrm{VO}_{2 s t}$ calculated for the different algorithms demonstrated that all of them provided $\mathrm{VO}_{2}$ data that were linearly affected by the changes of the stroke volumes of the "aberrant" cycles.

As could be expected, the "Mouth" algorithm was sensitive to all the "aberrant" cycles, and resulted in practically null "accounted" $\Delta \mathrm{VO}_{2 s t}$ in almost all the investigated conditions.

The EXP algorithm yielded different oxygen uptakes only when the "aberrant" part of the cycle involved the expiratory volume; it should be remembered here, however, that the EXP algorithm completely neglects the inspiratory phase, and consequently, also its perturbations. An increase in the stored oxygen volume showed an apparently complete compensation, while a depletion was overestimated of about $34 \%$.

The WES and IND algorithms yielded overlapping results; both algorithms provided oxygen uptakes practically equal to the "regular" cycles when the "aberrant" stroke was the expiratory one, whereas the $\mathrm{VO}_{2}$ values changed in the case the "aberrant" stroke was the inspiratory one. The correspondent "accounted" $\Delta \mathrm{VO}_{2 s t}$ calculated for the two algorithms were practically equal to the "theoretical" $\Delta \mathrm{VO}_{2 \mathrm{st}}$ when a depletion of the oxygen stores occurred, whereas their increase was underestimated of about $25 \%$.

Interestingly, in the case of an "aberrant" cycle where the same change involved first the inspiratory stroke and just thereafter the expiratory one (both in the same cycle), the calculated oxygen uptake was quite the same for all the algorithms, with changed values compared to the "regular" cycles. This result, however, should not surprise, since this type of cycle showed a changed ventilation with the same end-expiratory oxygen fraction, because the simulated expired gas was taken always from the same supply cylinder, independent of stroke volume; consequently, this condition had necessarily to result in a different $\mathrm{VO}_{2}$.

\subsection{Peculiarities of the used GESS}

The Gas Exchange Simulation Systems described in the literature (Boutellier et al., 1981; Bunn et al., 2011; Gore et al., 1997; Huszczuk et al., 1990; Prieur et al., 1998a) mainly pump only gases at ambient temperature, despite human expired air is warm and water vapor saturated. An exception is the GESS used by Prieur et al. (2003), that provided expired gas fully saturated with water vapor and at about $30{ }^{\circ} \mathrm{C}$, prepared in advance in a specific bag (Prieur et al., 1998b).

The GESS used in the present investigation allowed to heat and saturate the simulated expired gas, making it flow across a thermostatic water bath. This is an important feature, in particular when measurements are performed with a commercial metabolic unit, which software expects warm and saturated gas during the expiration phases. The thermostatic bath has been placed between the output of the syringe and the sensors of the metabolic unit. Water of the bath was set to $37^{\circ} \mathrm{C}$, although we were aware that the distance traveled by the flow (about $50 \mathrm{~cm}$ ) inevitably would result in a reduction of its temperature. Nevertheless, in volunteers exercising on a cycle ergometer $(100 \mathrm{~W}$ work load) the temperature of the expired gases, as measured at $1.5 \mathrm{~cm}$ from the mouth, was $33.6{ }^{\circ} \mathrm{C}$ (Hughson et al., 1991). Interestingly, the same authors also observed that the temperature of the expiratory flow had a significant effect on the oxygen uptakes calculated at the mouth, whereas the values obtained by the "alveolar" algorithms were not affected, because the nitrogen volume entered into the calculations. Similarly, we recalculated the oxygen uptakes hypothesizing that the temperature of the simulated expiratory flow could be in the range from $30^{\circ} \mathrm{C}$ to $40^{\circ} \mathrm{C}$. Fig. 6 shows that all the four investigated algorithms, including the "Mouth" one, provided overlapping values when the expiratory flow temperature was set to about $33^{\circ} \mathrm{C}$. Moreover, in agreement with Hughson et al. (1991), the temperature of the expiratory flow affected only marginally the oxygen uptake values obtained with the algorithms where the nitrogen volume is one of the variables within the equations (EXP, IND, and WES). According to these mathematical simulations, the difference in the average $\mathrm{VO}_{2}$ values between the "Mouth" algorithm and the other investigated algorithms (about $+0.055 \mathrm{~L} / \mathrm{min}$ ), although being in the range of the breath-by-breath fluctuations for exercising humans (Francescato and Cettolo, 2019), is likely explained also by the effects of the temperatures used in the calculations.

The GESS used in the present investigation keeps separated the gas

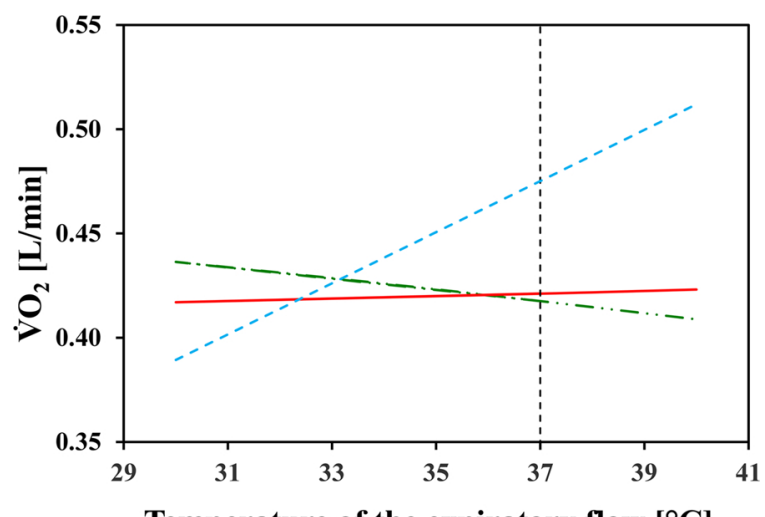

Temperature of the expiratory flow $\left[{ }^{\circ} \mathrm{C}\right]$

Fig. 6. Average oxygen uptake values for the "regular" cycles as calculated by means of the four algorithms under investigation when different temperatures were set for the expiratory flow.

The four algorithms provided overlapping oxygen uptake values for an expiratory flow temperature set to about $33^{\circ} \mathrm{C}$. The algorithms where nitrogen volume appears within an intrinsic integral (EXP, WES and IND) are quite insensitive to changes of the expiratory temperature. Data of the WES and IND algorithms overlap over the whole range of investigated temperatures. — — "Mouth", _ . . _ EXP, and _ WES or IND algorithms, respectively. 
volumes of the filling (simulating inspiration) and emptying (simulating expiration) of the two syringes, hindering the mixing of the gas volumes, and allowing to know the composition of the used gases. An example of the traces recorded with the GESS is illustrated in Fig. 2 (panel A) in comparison with traces obtained on a human performing light intensity exercise (panel B). Despite the similarities between the triplets of traces, human flow looks noisier, with inspiration and expiration showing an asymmetry either in the duration and in the behavior of the waveform; moreover, human gas fraction traces show a drift during the final part of the expirations, that does not appear during the emptying of the syringes. On the basis of these characteristics, the use of the traces generated by the GESS allowed a more robust identification of the start and end points of the cycles (necessary for the "Wessel" and the "Expiration-only" algorithms); conversely, it made more aleatory the identification of the times corresponding to the endtidal fractions, in turn necessary to define the cycles according to the "Independent breath" algorithm. As a consequence, results of the present work were obtained under more favourable conditions for the "Wessel" and "Expiration-only" algorithms as compared to the "Independent breath" one.

Finally, on the basis of the average measured ventilation (10.9 L/ min in BTPS conditions; Fig. 3) and of the difference between the ambient and the supply cylinder $\mathrm{O}_{2}$ fractions (i.e., 20.95-16.00\%), a theoretical $\mathrm{VO}_{2}$ of $0.444 \mathrm{~L} / \mathrm{min}$ (STPD) can be calculated for the "regular" cycles. This value is within the expected fluctuations of breath-bybreath variability for exercising humans (i.e. $>0.050 \mathrm{~L} / \mathrm{min}$ ) (Francescato and Cettolo, 2019).

\subsection{Strengths and limitations}

The strength of the present work is that the same flow and gas fractions traces have been used for the calculation of the oxygen uptakes with all the investigated algorithms. Consequently, whatever error or problem arising from the simulator and/or from the metabolic unit, used to perform the measurements, will have affected in the same way the results obtained by all the algorithms. To be noted that a widely recognized reference breath-by-breath gas exchange algorithm is lacking (Golja et al., 2018), and that the adequacy of the gold-standard validation method, based on Douglas bag-collection analysis, is not entirely error-free and has come to be questioned for breath-bybreath quality control purposes (Ward, 2018). An alternative would be selecting, a priori, one of the investigated algorithms as reference one. Nevertheless, there is no evidence in the literature supporting a better performance/effectiveness of one of them.

Main limitation of the present work is that only one stroke frequency with a fixed stroke volume was investigated for the "regular" cycles. Nevertheless, changes have been set to the stroke volume, either for the inspiratory one and/or for the expiratory one; although increased volumes were essentially set, overall the changes were in the range from $-0.3 \mathrm{~L}$ to $+1.5 \mathrm{~L}$, which is a considerable variation from the "regular" stroke volume of $1.0 \mathrm{~L}$. These "aberrant" strokes have induced changes in the oxygen volume stored in the system which have been calculated for differences with the "regular" cycles. Consequently, it would have been meaningless to repeat the measurements imposing the "aberrant" strokes on different volumes/frequencies applied to the "regular" strokes: nothing more than a few further points would be added on the already obtained regression lines.

Although we are aware that in vivo changes of the volume of oxygen stored in the lungs might be also the consequence of changes of the endexpiratory oxygen fraction (Auchincloss et al., 1966), with the used GESS it was technically not possible to switch, for only one predetermined cycle, to another bag containing a different gas mixture to simulate expiration. Consequently, it was not possible, with the used experimental setup, to generate "aberrant" cycles where the end-expiratory gas fraction was changed. A GESS designed to allow controlled changes of the end-expiratory gas fractions in the absence of a corresponding change of the pumped volumes, could be of interest to highlight the relevance of the fraction changes in determining the performance of the different algorithms when changes in the oxygen volume stored in the system occur. The separate analysis of the effects of "aberrant" cycles induced by changes of either pumped volume or end-respiratory fractions will allow to understand better what happens in humans, where the two phenomena occur contemporarily.

A further limitation is that only one gas mixture has been used to simulate the expiratory flow, which resulted in a respiratory quotient equal to 1 . However, the change of the alveolar volume due to a respiratory quotient in the range $0.83-1.3$ introduces a maximal error, empirically calculated, of $1.4 \%$ (Giezendanner et al., 1983), thus likely affecting only marginally the main results of the present work.

Finally, the algorithms requiring an optimization procedure after a first calculation of gas exchange, i.e., Swanson's algorithm (Swanson, 1980) and Busso and Robbins's algorithm (Busso and Robbins, 1997), were ignored, because they are not directly applicable on a metabolic unit, where real-time values are expected. The algorithm of Auchincloss et al. (Auchincloss et al., 1966; Beaver et al., 1981) was neglected as well, because the volume of the Functional Residual Capacity has no meaning for the GESS, while it is a required value in the calculations.

\section{Conclusions}

This is the first work that, using a GESS, investigated the performance of different algorithms for the calculation of breath-by-breath oxygen uptake when changes in oxygen stores were induced from one cycle to the next one.

Results show that the algorithms at stake (with the exception of the "Mouth" algorithm) had overall similar performances for the "regular" cycles, all of them being quite insensitive to the temperature set for the expiratory flow. This supports the conclusion that each one of these algorithms can be appropriately used under steady oxygen stores.

Conversely, when abrupt changes in the stored $\mathrm{O}_{2}$ volume were induced, the IND and WES algorithms were able to account for the changes more correctly as compared to the EXP algorithm. Consequently, when the oxygen stores change from on cycle to the following one, the use of the WES and/or IND algorithms should be recommended.

\section{Conflicts of interest}

None of the authors have conflicts of interest to declare.

\section{Acknowledgments}

Financial support by the Deutscher Akademischer Austauschdienst (DAAD) for the stay in Cologne (Germany) of MPF for the present investigation is acknowledged.

We thank the Cortex GmbH (Liepzig, Germany) company for having provided us with the metabolic unit. Cortex $\mathrm{GmbH}$, however, was not involved in the study design, data collection, analysis or interpretation.

This research did not receive any specific grant from funding agencies in the public, commercial, or not-for-profit sectors. 


\section{Appendix A}

This section summarizes, for the sake of convenience, the mathematical equations used in the present work for the different algorithms; the equations, as well as the underlying theory, have already been reported in previous papers (Cettolo and Francescato, 2018) to which the reader is referred to for further details.

Ventilation $\left(\dot{V}_{\mathrm{E}}\right)$ was calculated according to the following equation:

$\dot{\mathrm{V}} E_{j}=-\frac{\int_{t_{e, j}}^{t_{i, j}} \dot{V} \cdot d t}{t_{i, j}-t_{i, j-1}}$

where $\mathrm{V}$ is the respiratory flow; $t_{i, j}$ and $t_{e, j}$ are two successive time points where the measured flow changes orientation. According to its traditional description, a respiratory cycle starts at the onset of inspiration and ends when expiratory flow has entirely or largely ceased. Breath frequency $\mathrm{b}_{\mathrm{f}}$, breath/min) was then calculated as $60 /\left(t_{i, j}-t_{i, j-1}\right)$.

The difference between the volume of $\mathrm{O}_{2}$ introduced during inspiration and the volume of $\mathrm{O}_{2}$ exhaled during expiration for the $\mathrm{j}$-th breath (i.e., the "Mouth" algorithm) was calculated by simple integrating, over the whole breath, the instantaneous flow multiplied by the $\mathrm{O}_{2}$ fraction:

$\dot{\mathrm{VO}}_{2 \mathrm{j}} \mathrm{M}=-\frac{\int_{\mathrm{t}_{\mathrm{j}, j-1}}^{\mathrm{t}_{\mathrm{j}, \mathrm{j}}} \dot{\mathrm{V}} \cdot \mathrm{FO}_{2} \mathrm{dt}}{\mathrm{t}_{\mathrm{i}, \mathrm{j}}-\mathrm{t}_{\mathrm{i}, \mathrm{j}-1}}$.

where $\mathrm{FO}_{2}$ is the instantaneous oxygen fraction.

Making the assumption that only $\mathrm{O}_{2}$ and $\mathrm{CO}_{2}$ exchange occur at the alveolar-capillary membrane, and that consequently a negligible exchange occurs for all the other gases (overall considered as "nitrogen"), the $\mathrm{V}_{2}$ during the $\mathrm{j}$-th breath was calculated using information obtained only during expiration ("Expiration-only" algorithm) and applying the so-called Haldane transformation (Roecker et al., 2005; Ward, 2018):

$\dot{\mathrm{V}} \mathrm{O}_{2 j} \mathrm{EXP}=\frac{\int_{t_{e, j}}^{t_{i, j}} \dot{\mathrm{V}} \cdot \mathrm{FO}_{2} \mathrm{dt}-\frac{\mathrm{FIO}_{2}}{\mathrm{FIN}_{2}} \cdot \int_{\mathrm{t}_{\mathrm{e}, \mathrm{j}}}^{\mathrm{t}_{\mathrm{i}, \mathrm{j}}} \dot{\mathrm{V}} \cdot \mathrm{FN}_{2} \mathrm{dt}}{\mathrm{t}_{\mathrm{i}, \mathrm{j}}-\mathrm{t}_{\mathrm{i}, \mathrm{j}-1}}$

where $\mathrm{FN}_{2}$ is the instantaneous "nitrogen" fraction; $\mathrm{FIO}_{2}$ and $\mathrm{FIN}_{2}$ are the inspired ambient fractions and were set to $20.95 \%$ and $79.02 \%$, respectively.

Oxygen uptake at alveolar level was calculated according to "Wessel" algorithm (1979) using the following equation:

$\dot{V} O_{2 j}{ }^{W E S}=\frac{\int_{t_{i, j}-1}^{t_{i, j}} \dot{V} \cdot F O_{2} d t-\frac{F_{2}\left(t_{x, j}\right)}{F_{2}\left(t_{x, j}\right)} \cdot \int_{t_{i, j-1}}^{t_{i, j}} \dot{V} \cdot F N_{2} d t}{t_{i, j}-t_{i, j-1}}$

where $t_{x, j}$ is the time point corresponding to the end-expiratory gas fraction. The further assumption was made that the end-expiratory gas fractions reliably represent the "mixed" alveolar gas fractions (Auchincloss et al., 1966).

Finally, the following equation was used to calculate $\mathrm{O}_{2}$ uptake at alveolar level using the "Independent breath" algorithm (Cettolo and Francescato, 2018):

$\dot{\mathrm{V}} \mathrm{O}_{2 \mathrm{j}} \mathrm{IND}^{\mathrm{N}}=\frac{\int_{\mathrm{t}_{\mathrm{i}, \mathrm{j}}}^{\mathrm{t}_{2, \mathrm{j}}} \dot{\mathrm{V}} \cdot \mathrm{FO}_{2} \mathrm{dt}-\frac{\mathrm{FO}_{2 \mathrm{j}}\left(\mathrm{t}_{1, j}\right)}{\mathrm{FN}_{2 \mathrm{j}}\left(\mathrm{t}_{1, j}\right)} \cdot \int_{\mathrm{t}_{1, j}}^{\mathrm{t}_{2, j}} \dot{\mathrm{V}} \cdot \mathrm{FN}_{2} \mathrm{dt}}{\mathrm{t}_{2, \mathrm{j}}-\mathrm{t}_{1, \mathrm{j}}}$

where the start and end point of the respiratory cycle $\left(t_{1, j}\right.$ and $t_{2, j}$, respectively) are identified on two consecutive expirations as the time points where identical ratios between $\mathrm{FO}_{2}$ and $\mathrm{FN}_{2}$ are observed (Cettolo and Francescato, 2015).

\section{References}

Auchincloss, J.H.J., Gilbert, R., Baule, G.H., 1966. Effect of ventilation on oxygen transfer during early exercise. J. Appl. Physiol. 21, 810-818.

Beaver, W.L., Wasserman, K., Whipp, B.J., 1973. On-line computer analysis and breath by-breath graphical display of exercise function tests. J. Appl. Physiol. 34, 128-132.

Beaver, W.L., Lamarra, N., Wasserman, K, 1981. Breath-by-breath measurement of true alveolar gas exchange. J. Appl. Physiol. 51, 1662-1675.

Boutellier, U., Gomez, U., Mader, G., 1981. A piston pump for respiration simulation. J. Appl. Physiol. 50, 663-664.

Bunn, J.A., Pittsley, J.L., Baker, S.V., Yates, J., 2011. Assessment of accuracy of the VacuMed 17053 calibrator for ventilation, oxygen uptake $(\mathrm{V}(\mathrm{O}(2)))$, and carbon dioxide production (V(CO(2))). Respir. Care 56, 472-476. https://doi.org/10.4187/respcare. 00951.

Busso, T., Robbins, P.A., 1997. Evaluation of estimates of alveolar gas exchange by using a tidally ventilated nonhomogenous lung model. J. Appl. Physiol. 82, 1963-1971.

Cettolo, V., Francescato, M.P., 2015. Assessment of breath-by-breath alveolar gas exchange: an alternative view of the respiratory cycle. Eur. J. Appl. Physiol. 115, 1897-1904. https://doi.org/10.1007/s00421-015-3169-x.

Cettolo, V., Francescato, M.P., 2018. Assessing breath-by-breath alveolar gas exchange: is the contiguity in time of breaths mandatory? Eur. J. Appl. Physiol. 118, 1119-1130. https://doi.org/10.1007/s00421-018-3842-y.

Francescato, M.P., Cettolo, V., 2019. The "independent breath" algorithm: assessment of oxygen uptake during exercise. Eur. J. Appl. Physiol. 119, 495-508 doi: 10/1007/ s00421.018.4046-1.

Giezendanner, D., Cerretelli, P., Di Prampero, P.E., 1983. Breath-by-breath alveolar gas exchange. J. Appl. Physiol. 55, 583-590.

Golja, P., Cettolo, V., Francescato, M.P., 2018. Calculation algorithms for breath-bybreath alveolar gas exchange: the unknowns!. Eur. J. Appl. Physiol. 118, 1869-1876. https://doi.org/10.1007/s00421-018-3914-Z.

Gore, C.J., Catcheside, P.G., French, S.N., Bennett, J.M., Laforgia, J., 1997. Automated' VO2max calibrator for open-circuit indirect calorimetry systems. Med. Sci. Sports Exerc. 29, 1095-1103.

Grønlund, J., 1984. A new method for breath-to-breath determination of oxygen flux across the alveolar membrane. Eur. J. Appl. Physiol. 52, 167-172.

Hughson, R., Northey, D., Xing, H., Dietrich, B., Cochrane, J., 1991. Alignment of ventilation and gas fraction for breath-by-breath respiratory gas exchange calculations in exercise. Comput. Biomed. Res. 24, 118-128.

Huszczuk, A., Whipp, B.J., Wasserman, K., 1990. A respiratory gas exchange simulator for routine calibration in metabolic studies. Eur. Respir. J, 3, 465-468.

Prieur, F., Busso, T., Castells, J., Bonnefoy, R., Benoit, H., Geyssant, A., Denis, C., 1998a. A system to simulate gas exchange in humans to control quality of metabolic measurements. Eur. J. Appl. Physiol. 78, 549-554. 
Prieur, F., Busso, T., Castells, J., Bonnefoy, R., Benoit, H., Geyssant, A., Denis, C., 1998b. Validity of oxygen uptake measurements during exercise under moderate hyperoxia. Med. Sci. Sports Exerc. 30, 958-962.

Prieur, F., Castells, J., Denis, C., 2003. A methodology to assess the accuracy of a portable metabolic system (VmaxST). Med. Sci. Sports Exerc. 35, 879-885. https://doi.org/10. 1249/01.MSS.0000065003.82941.B0.

Rodriguez, F.A., Keskinen, K.L., Kusch, M., Hoffmann, U., 2008. Validity of a swimming snorkel for metabolic testing. Int. J. Sports Med. 29, 120-128. https://doi.org/10. 1055/s-2007-964973.

Roecker, K., Prettin, S., Sorichter, S., 2005. Gas exchange measurements with high temporal resolution: the breath-by-breath approach. Int. J. Sports Med. 26, S11-S18. Swanson, G., 1980. Breath-to breath considerations for gas exchange kinetics. In:

Cerretelli, P., Whipp, B.J. (Eds.), Exercise Bioenergetics and Gas Exchange. Elsevier/ North Holland, Amsterdam, pp. 211-222.

Ward, S.A., 2018. Open-circuit respirometry: real-time, laboratory-based systems. Eur. J. Appl. Physiol. https://doi.org/10.1007/s00421-018-3860-9.

Wessel, H., Stout, R., Bastanier, C., Paul, M., 1979. Breath-by-breath variation of FRC: effect on $\mathrm{VO}_{2}$ and $\mathrm{VCO}_{2}$ measured at the mouth. J. Appl. Physiol. Respir. Environ. Exerc. Physiol. 46, 1122-1126. 